rate of PID following gonorrhoea infection. This analysis compares the rate of hospitalisation for PID following a diagnosis of gonorrhoea to the rate following a chlamydia diagnosis.

Method All women, aged 15-45 years, in New South Wales (NSW), with a diagnosis of chlamydia or gonorrhoea between $1 / 7 / 2000$ and $31 / 12 / 2008$ were followed for up to one year after diagnosis for hospitalisations for PID. The incidence rates of PID hospitalisation among women with a chlamydia or gonorrhoea diagnosis were compared to the whole of the NSW population using standardised incidence ratios (SIRs). Poisson regression was used to compare the rates of PID hospitalisation after adjusting for age, diagnosis date, socioeconomic group, area of residence and prior births.

Results There were 38379 women with a chlamydia diagnosis. During 35014 person years of follow-up (PYFU), 485 were hospitalised for PID; incidence rate (IR) 13.8 per 1000 PYFU (95\% CI 12.615.1). Among 1023 women with a gonorrhoea diagnosis, during 895 PYFU 45 were hospitalised for PID; (IR 50.3 per 1000 PYFU, 95\% CI 35.6-65.0). Compared to the age-equivalent NSW female population, the incidence of PID hospitalisation was 27.0 (95\% CI 24.429.8) times greater among women who had a chlamydia diagnosis in the year prior to hospitalisation and 95.8 (95\% CI 64.2-137.6) times greater among women with a gonorrhoea diagnosis. Younger age, diagnosis prior to 2005, socioeconomic disadvantage and prior births were also associated with an increased rate of PID hospitalisation.

Conclusion Hospitalisation rates for PID were over 3 times greater in women diagnosed with gonorrhoea than chlamydia, and rates in both were substantially higher than in the general female population. Our results suggest that gonorrhoea causes more serious reproductive health sequelae than chlamydia.

\section{P3.011 DRY SWAB EVALUATION BY ROCHE 4800 CT/NG AND THE PRESTO-PLUS: CROSS-SECTIONAL STUDY OF GENITAL, RECTAL AND PHARYNGEAL CHLAMYDIA AND GONORRHOEA INFECTION IN WOMEN IN RURAL SOUTH AFRICA}

doi:10.1136/sextrans-2013-051184.0471

'D J de Waaij, ${ }^{1,2}$ J Dubbink, ${ }^{1,2} \mathrm{~L}$ van der Eem, ' $\mathrm{M}$ L A Bos, 'S Ouburg, ${ }^{3,4} \mathrm{D}$ A Lewis, ${ }^{2} \mathrm{H}$ Struthers, $2,5 \mathrm{~J}$ A McIntyre, 1,6S A Morré, ${ }^{2} \mathrm{R}$ P H Peters. ' VU University Medical Center, Amsterdam, The Netherlands; ${ }^{2}$ Anova Health Institute, Johannesburg and Tzaneen, South Africa; ${ }^{3}$ National Institute for Communicable Diseases, National Health Laboratory Service, Johannesburg, South Africa; ${ }^{4}$ University of the Witwatersrand, Johannesburg, South Africa; ${ }^{5}$ University of Cape Town, Cape Town, South Africa; ${ }^{6}$ University of Maastricht, Maastricht, The Netherlands

Backgound Epidemiological data, required to inform the design and implementation of control programmes, in relation to genital Chlamydia and Gonorrhoea in rural Africa, are limited. There are no data on the prevalence of rectal or pharyngeal infections among women. We evaluate the use of dry swabs by Roche 4800 CT/NG and the PRESTO-PLUS to determine the prevalence in three anatomic locations of chlamydial and gonococcal infections in African women.

Methods Cross-sectional study of 604 women visiting 25 randomly selected primary healthcare (PHC) facilities. Participants were recruited between November 2011 and February 2012. Setting: PHC facilities across rural Mopani District in Limpopo Province, South Africa. Participants: Women aged 18-49 years who reported at least one sex act in the past 6 months were eligible. A questionnaire was administered and physical examination conducted. Vaginal, anorectal and oropharyngeal swabs were tested for Chlamydia trachomatis and Neisseria gonorrhoeae.

Results 480 (including 5 times 4 controls) samples have been tested by both assays at this moment, the others are in progress. Overall prevalence of either infection was $20 \%$. Prevalence of genital chlamydia was $13 \%$ and gonorrhoea $5 \%$; rectal chlamydial infection was diagnosed in $4 \%$ and gonococcal in $2 \%$ of women. Clear geographical differences were observed in the CT and NG prevalances. Roche and PRESTO-PLUS had similar prevalences with slightly higher prevalances found by PRESTO-PLUS, however not all 604 samples have been tested, and discrepancy analyses will be performed in the upcoming 2-3 months.

Conclusion Dry swab collection seems a reliable method of sampling without majot prevalence differences between Roche and PRESTO-PLUS. Genital and rectal, but not pharyngeal, chlamydia and gonorrhoea infections are highly prevalent and frequently asymptomatic in women in rural South Africa. Young women attending healthcare facilities for antenatal care or family planning should be prioritised in control efforts.

\section{P3.012 HOW ROBUST ARE THE DESCRIPTIONS OF CHLAMYDIA NATURAL HISTORY USED IN ECONOMIC EVALUATIONS OF CONTROL STRATEGIES?}

doi:10.1136/sextrans-2013-051184.0472

'B Davies, 'S Anderson, ${ }^{2} \mathrm{~K}$ M E Turner, 'H Ward. 'Imperial College London, London, UK; ${ }^{2}$ University of Bristol, Bristol, UK

Background The decision to implement a Chlamydia screening programme is based on a detailed assessment of its projected impact and cost-effectiveness. In the absence of evidence from randomised controlled trials, transmission dynamic models are crucial to this process. However these models are highly sensitive to the representation of the infection. We review the evidence used to inform the model parameters highlighting their strengths and limitations.

Methods Published economic analyses of chlamydia screening interventions were identified following a systematic search of the literature. Only transmission dynamic models were included as they represent the gold standard. Parameters describing chlamydia infection were extracted and the variability across the studies assessed. The data used to inform each parameter was sourced and critically evaluated.

Results Eleven studies were included in this review, all evaluating chlamydia screening programme designs in developed countries. Many key natural history parameters are based on sparse historical data and there is wide variation in the values used across the models. For example,

- The per act transmission probability ranging from $3.75 \%$ to $15.3 \%$.

- The modelled duration of asymptomatic infection was between 180-370 days in women and 40-200 days in men.

- Only one paper includes a period of protective immunity following infection.

- Only 2 studies consider the role of reinfection in the development of complications

- However, there is a general consensus in the proportion of people that are asymptomatic; between $70-75 \%$ of women and $25-50 \%$ of men.

Conclusion We highlight the variability in descriptions of the natural history and emphasise the importance of using contemporary data to inform modelling studies. A clear consensus on the appropriate representation of the natural history is needed, with estimates continuously updated using new evidence.

\section{P3.013 THE ROLE OF CHLAMYDIA IN PELVIC INFLAMMATORY DISEASE AND ECTOPIC PREGNANCY}

doi:10.1136/sextrans-2013-051184.0473

${ }^{1} \mathbf{M}$ Price, ${ }^{2} \mathrm{~A}$ Ades, ${ }^{2} \mathrm{~N}$ Welton, ${ }^{3} \mathrm{~K}$ Soldan, ${ }^{4} \mathrm{P}$ Horner. ${ }^{1}$ University of Birmingham, Birmingham, UK; '2University of Bristol, Bristol, UK; ${ }^{3}$ Health Protection Agency, London, UK; ${ }^{4}$ University of Brisol, Bristol, UK 